\title{
An Adaptive Logical Link Control For Wireless Internet Service in ITS
}

\author{
Chi-Hyun Park and Dong-Ho Cho \\ Communication and Information Systems Laboratory \\ Department of Electrical Engineering \\ Korea Advanced Institute of Science and Technology \\ E-mail: chihyun@comis.kaist.ac.kr, dhcho@ee.kaist.ac.kr
}

Abstract DSRC(Dedicated Short Range Communications), which is a radio mobile communication protocol in ITS, is suited for traffic related services. Considering the current trends to introduce wireless Internet service into the mobile communication network, wireless Internet service in ITS system is valuable. An adaptive logical link control algorithm to support wireless Internet web service in DSRC based on the reliable high speed data transmission is proposed and it is shown that an enhanced performance could be obtained compared with the DSRC logical link control algorithms proposed by Europe and Japan.

\section{Introduction}

ITS (Intelligent Transportation System) is a next generation transportation system, which aims to increase efficiency, convenience and traffic safety with improvement of infrastructures and vehicles. The way to exchange information between computer centers and vehicles is one of the main targets in ITS. There are two radio communication types in mobile communication: wide-area wireless communications (WAC) and dedicated short-range communications (DSRC). For ITS services, it is preferable for DSRC to be mainly used in view of efficiency. But for the smooth growth of ITS, we should consider the share of WAC and DSRC in view of applications of ITS. Especially considering the current trends to introduce wireless Internet service into the mobile communication network, wireless Internet service is valuable to ITS services.

The international pre-standards for DSRC are composed of the specification for three layers. The small service areas and critical real-time constraints require a specific protocol architecture leading to reduced protocol stack, built up by the physical layer, data link layer and application layer. LLC (Logical Link Control), sub-layer of data link layer, defines LPDU transfer and error control on wireless link between vehicle and roadside equipment (computer center). Although ITS LLC protocols recommended by Europe and Japan are effective for services related with traffic information, it is not suitable for services related with geographical information, image or Internet. That is the reason the data traffic characteristics is changed according to service types. DSRC service data such as traffic information, location information, speed information, and traffic management information are very often transmitted or exchanged on the basis of about 64-byte frame. Other service data such as geographical information, image, and Internet web data have long length frame and have the unsymmetrical data transmission characteristics between up and down link. That is, down link traffic is generated much more than up link traffic. For Internet web service, service request data is generally about 320 byte length and Internet web service data is about $8 \sim 10 \mathrm{Kbyte}$ length.

Therefore, we suggest a logical link control algorithm effective for Internet web service as well as traffic related services, and analyze its performance improvements. For traffic related service, the performance of DSRC LLC recommended by Europe and Japan is the same as that of proposed LLC protocol. However, for wireless Internet web service, the performance of proposed protocol is better than that of LLC protocol used in Europe and Japan.

The rest of this paper is organized as follows: In Section 2, we present LLC protocols proposed by Europe and Japan. In Section 3, we describe our proposed LLC protocol, adaptive logical link control 
protocol, and in Section 4, we analyze the performance of each protocol, LLC Type3 recommended by Europe and Japan and LLC Type 4 proposed by us, and compare the analysis results. Finally, conclusions are made in Section 5.

\section{DSRC LLC protocol}

\subsection{DSRC LLC protocol overview}

The LLC sub-layer provides a description of the peer-to-peer protocol that is defined for the transfer of information and control between a pair of data link service access points in a DSRC communication environments. Each DSRC LLC protocol recommended by Europe and Japan provides two types of operation such as unacknowledged connectionless mode called LLC Type1 and acknowledged connectionless mode called LLC Type3. Only Type 3 supports the error control and sequence delivery. Type 3 uses stop-and-wait ARQ that is suitable for DSRC services of which data length is very short.

Fig. 1 shows the LPDU format that consists of Control field and Information field and Fig.2 shows Control field.

\begin{tabular}{|c|c|}
\hline Control field & Information field (LSDU) \\
$\leftarrow \leftarrow \quad 1$ byte $\rightarrow \mid \leftarrow 8 \times M$ bytes, $\quad M>=0$, integer $\rightarrow \mid$
\end{tabular}

Fig.1 LLC PDU Format

\begin{tabular}{|l|l|l|l|l|l|l|l|}
\hline 1 & 1 & M1 & M2 & P/F & M3 & M4 & M5 \\
Mn $:$ Modification function bit \\
P/F : Poll/Final bit
\end{tabular}

Fig.2 LPDU Control Field of Type3

The one-byte control field is used to provide data link control functions and information transfer. This field contains a $\mathrm{P} / \mathrm{F}$ bit corresponding to command / response and modifier function bit. For Type3 operation, the P/F and M5 bit of Control field are set by Stop-and-Wait ARQ and M1 M4 bits are set to
"1011". Information field consists of any integer number of octets. For response LPDU, this field is divided into two sub-fields. Each of them is one- byte status sub-field and LSDU sub-field, which are shown in Fig.3.

\begin{tabular}{|l|c|}
\hline Status sub-field & LSDU ( Data Sub-field) \\
\hline$\leftarrow \quad 1$ byte $\rightarrow \mid \leftarrow 8 \times(\mathrm{M}-1)$ bytes, $M>=0$ (정수) $\rightarrow \mid$
\end{tabular}

Fig.3 Response Information Field

In the case of Type 3 operation, the sending LLC transmits command PDU of which $\mathrm{P} / \mathrm{F}$ bit is set to $\mathrm{P}$ and starts timer and increments an internal transmission count variable. If no response PDU is received before the timer expires, the sending LLC retransmits the command, increments the internal transmission count variable, and reset and restart timer. These retransmission procedures are repeated until the value of the internal transmission count variable is equal to the maximum number of retransmission. On the other hand, on receipt of an command, the receiving LLC may acknowledge with response $\mathrm{PDU}$ of which $\mathrm{P} / \mathrm{F}$ bit is set to $\mathrm{F}$, and M5 bit is set to the complement of M5 of received command PDU and Information field contains only status subfield. In summary, LLC protocol uses Stop-and-Wait ARQ that is effective for transmitting very short data, especially data encapsulated in one LPDU. Generally, DSRC service data such as traffic information, location information, speed information, and traffic management information are very often transmitted or exchanged on the basis of about 64-byte frame. As a result, when the length of LSDU is larger than the length of traffic related data, the performance of LLC is good and this case can occur in ITS services.

2.2 Performance Analysis of DSRC LLC Type3 protocol

The performance of LLC protocol can be measured by throughput. The following equation defines throughput.

$$
S=\left(N M_{L P D U} \times T_{L S D U}\right) \div \text { Total-time }
$$

$\mathrm{NUM}_{\text {LPDU }} \quad:$ the total number of transmitted LPDUs $\mathrm{T}_{\text {LSDU }}:$ the time needed for transferring a LSDU

Total-time : the total time needed for transferring NUM $_{\text {LPDU }} \times$ LPDUs successfully.

We suppose that the length of LPDU is longer than 
the length of Type 3 service data because Type3 service data is very short. Thus throughput of Type 3 represents the ratio of the time for transferring one LSDU to $E\left[T_{1}\right]$, the expected time for transferring one LPDU successfully. Thus we have

$$
\begin{aligned}
E\left[T_{1}\right] & =\left(1-P_{L P D U}\right)\left\{\left(t_{L P D U}+t_{a c k}\right)\left(1-P_{\text {ack }}\right)+\left(t_{L P D U}+t_{\text {timer }}+T_{1}\right) P_{a c k 3}\right\} \\
& +P_{L P D I}\left(t_{L P D U U}+t_{\text {timer }}+T_{1}\right)
\end{aligned}
$$

Each item in (2) is defined in (3). $E\left[T_{1}\right]$ results from the sum of one LPDU transfer time multiplied by failure rate and success rate separately. Each one LPDU transfer time is calculated by Type 3 procedure, considering an error probability in both command PDU and response PDU.

\section{Adaptive LLC protocol}

\subsection{LLC Type4 protocol overview}

We design a logical link control algorithm which provides error and flow control mechanism to support the reliable high speed data transmission in wireless link between roadside equipment and vehicle. At the same time, we consider that it operates adaptively to the service type such as traffic related service and Internet web service. We will refer this protocol to LLC Type 4. The algorithm and the frame format for adaptive LLC are described below.

The LPDU is composed of two fields, control field and data information field each of which is the same size and structures of DSRC LLC in Europe and Japan. Only difference is the control field elements which consist of EF (End Flag - 1bit), Window Size ( 2 bits), Sequence number ( 3 bits) and $C / R$ (command/Response - 1bit).

\begin{tabular}{|l|l|l|l|l|l|l|l|}
\hline EF & M & W1 & W2 & P/F & S1 & S2 & S3 \\
\hline
\end{tabular}

$$
\begin{aligned}
& \text { EF : End Flag } \\
& \text { M : Modifier Function bit } \\
& \text { W1, W2 : Window size } \\
& \text { P/F : Poll/Final bit } \\
& \text { S1, S2, S3 : Sequence number }
\end{aligned}
$$

Fig.4 Type4 Control field

The sender starts data transmission by setting the window size, sequence number, $\mathrm{C} / \mathrm{R}$ and $\mathrm{EF}$ bit. Window size field has 2-bit composition, 00(0), $01(1), 10(2)$, and 11(3), each of which means window size, $2^{0}, 2^{1}, 2^{2}$, and $2^{3}$. C/R bit is set by the same procedure of DSRC LLC. EF bit is set to '1' only when the last data frame in one window is transmitted. Sequence number is calculated by modulo 8 operation. After sending the last data frame in one window, the sender starts the timer to receive the acknowledgment from the receiver.

After the receiver accepts data frame, the status vector, that is composed of 8 bits each of which corresponds to the received sequence number is set until the data frame with $\mathrm{EF}$ set to ' 1 ' is received. After receiving the last data frame in one window size, the receiver transmits the data frame whose control field's window size is the same as that of the received data frame. Other procedures except these operations are the same as DSRC LLC.

When receiving the acknowledgement, the sender examines the Status sub-field of Information field and retransmits the data frame whose corresponding status bit is set to 'incorrect'. If the timer expires before the acknowledgment arrives, the sender requests the receiver to retransmit the acknowledgment and starts the timer for receiving acknowledgment. If the acknowledgement is not received after this procedure, the sender restarts transmitting data.

Thus proposed algorithm has the following merits. For traffic related service, its window size is almost 1, so throughput is the same as the DSRC LLC. For Internet web service, throughput is improved because data frames are transmitted through large window and acknowledged by one unit window.

\subsection{Performance Analysis of DSRC LLC Type4 protocol}

We suppose that the length of LPDU is longer than that of Type 3 service data. Because LLC Type4 procedure is different according to the window size, we can calculate throughput as follows.

$$
\begin{aligned}
& \text { i) } 1<\text { i }<=\text { window size }
\end{aligned}
$$

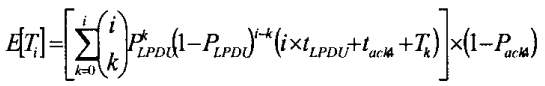

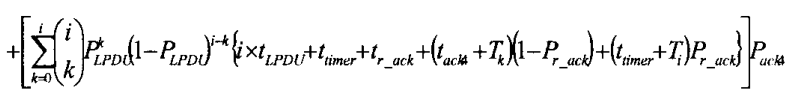


ii) $\mathrm{i}=1$

$$
\begin{aligned}
E\left[T_{1}\right] & =\left(1-P_{L P D U}\right)\left\{\left(t_{L P D U}+t_{a c k 4}\right)\left(1-P_{a c k 4}\right)+\left(t_{L P D U}+t_{\text {timer }}+T_{1}\right) P_{a c k 4}\right\} \\
& +P_{L P D U}\left(t_{L P D U}+t_{\text {timer }}+T_{1}\right)
\end{aligned}
$$

$\mathrm{P}_{\mathrm{LPDU}}=1-(1-\mathrm{BER})^{\mathrm{LPDU} \text { length }}$

: error rate of a command LPDU

$\mathrm{P}_{\text {ack3 }}, \mathrm{P}_{\text {ack4 }}$ : error rate of a Type3, Type4 response LPDU

$\mathrm{P}_{\mathrm{r} \text { ack }}$ : error rate of a command LPDU which requests the receiver to transmit a Type4 response LPDU $t_{L P D U}$ : the time needed for transferring a command LPDU

$t_{\text {ack3 }}, t_{\text {ack4 }}$ : the time needed for transferring a Type3, Type 4 response LPDU

$\mathrm{t}_{\mathrm{r}_{\mathrm{a}} \mathrm{cck}}$ : the time needed for transferring retransmit request $\mathrm{LPDU}$

$t_{\text {timer }}:$ timer expiration time

$T_{k}, T_{i}$ : the time needed for transferring $k$, i LPDUs

When the window size is 1 , Type 4 LLC operates as LLC Type 3. So $E\left[T_{1}\right]$, the expected time needed for transferring a Type 4 command LPDU is the same as that of Type 3.

On the other hand, we can calculate the time for transferring $\mathrm{i}$ ( $\mathrm{i}<=$ window size)LPDUs by the Type4 LLC operation when window size is bigger than 1 , that is, 2,4 or 8 . In this case, $\mathrm{E}\left[\mathrm{T}_{\mathrm{i}}\right]$ is derived from multiplying the error probability that may occur in $k$ PDUs of all $i$ command PDUs by the corresponding transmission time, changing $\mathrm{k}$ from 0 to $i$. At the same time, the error probability in response PDUs and response retransmit request PDUs are considered.

\section{Numerical Results and Discussion}

We analyzed the performance of two types of LLC protocol, such as Type 3 and Type 4 in view of throughput. In this section, we show the simulation results of both LLC protocols for internet web service. For Internet web service, the length of service data is $8 \sim 10$ Kbytes and this service data can be transferred through about 166 LPUDs. Because Type 4 operates as Type 3 for traffic related service, we analyzed the performance of these protocols only for internet web traffic. Fig. 5 shows the simulation results of type 3 and type 4 LLC protocols for internet web service.
The traffic model for internet web service has the distributions of ON and OFF periods with Weibull and Pareto with parameter $\left(\mathrm{k}=0.91 \sim 0.77, \theta=\mathrm{e}^{4.4} \sim\right.$ $\left.\mathrm{e}^{4.6}\right)$ and $(\alpha=0.9 \sim 0.58)$, respectively [4]. The interarrival time of requests within an ON period is governed by another Weibull distribution with parameters $k=0.5$ and $\theta=1.5$. The document size distribution is also the Pareto distribution of $\alpha<1$.

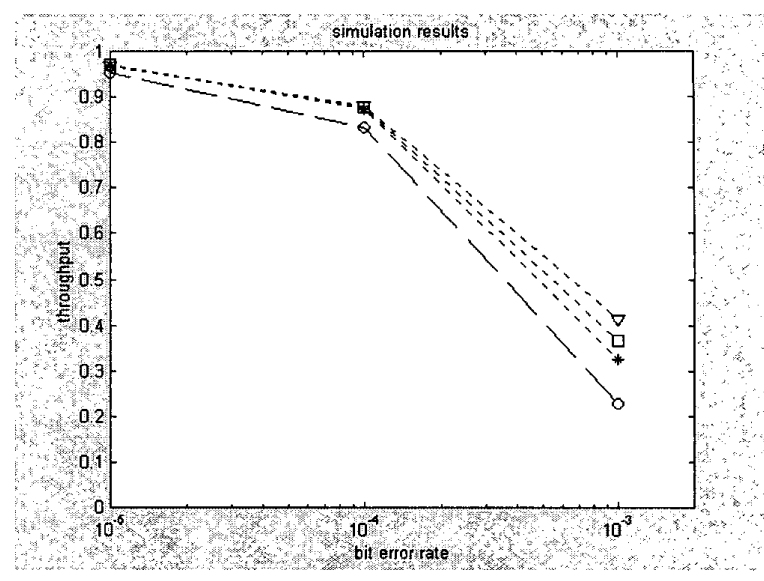

\begin{tabular}{|l|l|l|l|}
\hline $\begin{array}{l}\text { Type3 } \\
\text { Type4(WS =1) }\end{array}$ & $\begin{array}{l}\text { Type4 } \\
\text { WS }=8\end{array}$ & $\begin{array}{l}\text { Type4 } \\
\text { WS }=4\end{array}$ & $\begin{array}{l}\text { Type 4 } \\
\text { WS }\end{array}$ \\
\hline O --- & $* \ldots \ldots \ldots \ldots . .$. & $\square \cdots \cdots \cdots \cdots$ & $\nabla$ \\
\hline
\end{tabular}

Fig. 6 Simulation Results for Internet web service

As a result of this simulation, we show that the performance of LLC Type 4 is better than that of LLC Type 3. Then, the performance is changed with window size.

The effect of window size is as follows. For low BER on the wireless link, as window size increases, better performance could be obtained. But, as $\mathrm{BER}$ (bit error rate) on the channel is increased, performance can be enhanced with small window. The optimal window size is 2 in this simulation at $\mathrm{BER}=10^{-3}$.

The duration of timer expiration is also an important factor in performance improvement. For Type 3 LLC, there is one timer and for type 4 LLC, three timers. The duration of timer expiration is changed according to the purpose of the corresponding timer. Type 3 and Type 4 LLC protocols whose window size is 1 permit piggybacking. So, the timer duration for receiving the response PDU needs at least two times command PDU transmission time. When window size is 2,4 , or 8 , we need two timers, one for 
waiting acknowledgement after transmitting the last data and the other for waiting acknowledgement after requesting the receiver to retransmit acknowledgement. The first timer needs at least the time for transmitting both one command PDU and one response because piggybacking is not permitted for window whose size is bigger than 1 . The second timer needs at least the duration for transmitting two response PDUs.

In summary, Type 4 LLC enhances the performance of data link layer protocol in view of internet web service.

\section{Conclusions}

We proposed an adaptive logical link control protocol to provide both traffic related services and Internet web service efficiently in ITS and analyzed the performance of both proposed and DSRC LLC protocol recommended by Europe and Japan. According to the performance analysis, proposed protocol shows an performance improvement in view of supporting both traffic related services and Internet web service at the same time in ITS.

The proposed protocol is focused on non-real time service, so research on an adaptive logical link control algorithm suitable for both non-real time and real time service will be continued.

\section{References}

[1] The role of DSRC in ITS, Y.Kanazaki, T.Sasamoto, and H.Hirata, ITS World Congress, Berlin, Germany, Oct. 1997.

[2] "Transport Information and Control System(TICS)Dedicated Short Range Communication(DSRC)-Data Link Layer : Logical Link Control ver. 2.0", p. 83 121, ISO/TC204/WG15 Committee of Japan October 1997.

[3] William Stallings, "Data and computer communications", p. $164 \sim 197$, fourth ed.

[4] Shung deng, "Empirical model of WWW document arrivals at access link", ICC'96, p.1797 1802, 1996.

[5] GSM, "Radio link protocol", August 1997.

[6] TR45, "Radio link protocol", May 1997.

[7] D.N.Serpanos, J.R. Brunheroto and J.Nogima, "Data link control emulation: Rapid prototyping for highspeed networks", IEEE Network, p.56 64, March/April 1998. 